\title{
Investigation of corrosion defects in titanium by positron annihilation
}

Ryszard Pietrzak, Roman Szatanik

Abstract. The positron annihilation method was used to study the formation of defects in titanium samples during their corrosion in the vapor of a $3 \% \mathrm{HCl}$ solution. In particular, the distribution of defects depending on the distance from the corroding surface and the impact of an external magnetic field on the concentration of vacancies forming during the corrosion of titanium layers close to the surface were determined.

Key words: positron annihilation spectroscopy $\bullet$ corrosion defects

R. Pietrzak, R. Szatanik ${ }^{\bowtie}$

Institute of Physics,

Opole University,

48 Oleska Str., 45-052 Opole, Poland,

Tel.: +48 77452 7250, Fax: +48 77452 7290,

E-mail:szata@uni.opole.pl

Received: 19 June 2015

Accepted: 1 September 2015

\section{Introduction}

Positrons which penetrate substances annihilate with electrons in several ways. For instance, in defected metals, positron annihilation occurs either in the interstitial space or in the defects of the structure. In metals covered with a thin passive layer, positrons annihilate both in this layer and the defected volume of the metal. Admittedly, in Ti, the passive layer is of the type $n$, still cation vacancies that diffuse inside the metal occur in it also. Thus, the positron lifetime spectrum for Ti covered with a passive layer of its oxides contains, in itself, information on the sizes and concentrations of these defects. In systems, where only one type of defects exists, it is the so-called two-state positron annihilation model [1] that is used to interpret experimental results. With its help, it is possible to determine, among others, the mean concentration $C_{v}$ of defects:

$$
C_{v}=\frac{1}{v_{v}} \frac{I_{2}}{I_{1}}\left(\frac{1}{\tau_{B}}-\frac{1}{\tau_{2}}\right)
$$

where $\tau_{B}$ is the positron lifetime in bulk, $\tau_{2}$ is the value of the second component of positron lifetime spectra, $I_{1}$ and $I_{2}$ are the intensities of the first and second component of positron lifetime spectra, respectively, $v_{v}$ is the specific positron trapping rate for defect and vacancy-like defect in metals that usually falls into the range $10^{14}-10^{15} \mathrm{~s}^{-1}$. Note that the quality

$$
\tau_{f}=\left(I_{1} \tau_{1}^{-1}+I_{2} \tau_{2}^{-1}\right)^{-1}
$$

was evaluated and found to agree well with the Ti bulk is lifetime $\tau_{B}$.

Corrosion of metals is associated with the formation of lattice defects on the surface of the metal. 
Publications [2-4] demonstrate that the positron lifetime measurement technique can be useful for examinations of defects caused by chemical processes on the surface of the metal (e.g., corrosion). One aspect of corrosion that is not fully explained before is the effect of a magnetic field on the kinetics of this process [5-7]. Researchers have found, among other things, that the magnetic field strongly influences the kinetics of the formation of corrosion pits in ferromagnetic materials but have no contribution to the corrosion in austenitic steel grades. Our research has confirmed this effect $[4$, $8]$. The so-called point defect model (PDM) of corrosion [9] describes the huge role of lattice defects in the process of transferring cations outside and anions into the metal. Linhardt and coworkers [10] demonstrated that as long as the metal is passive, the magnetic field has no impact on the kinetics of corrosion pit formation in AISI 304 austenitic steel, but once the passive layer is broken or disappears, the magnetic field influences the rate at which pits are formed. The passivating, protective oxide layer is a two-phase area with a strong electrical field inside, in which cation vacancies of the metal move deeper into the metal, and oxide anion vacancies toward the sample surface. One could think that the passive layer on the surface of titanium behaves like a conductor through which electricity flows. According to the laws of electrodynamics, if this conductor is surrounded by a magnetic field, the Lorentz force acts on it, and the value of this force depends on the thickness of the passive layer and orientation of the external magnetic field relative to the electrical field in the passive layer.

This publication presents the results of the studies of the density distribution of corrosion defects in the near-surface layer of titanium samples as well as the effect of the magnetic field on the concentration of vacancies formed by the corrosion process in these samples.

\section{Preparation of samples, experimental procedure}

The titanium samples were made by Goodfellow, had the purity of $99.99 \%$, and were cylindrical with $10 \mathrm{~mm}$ in diameter and $2 \mathrm{~mm}$ in thickness. In order to eliminate above-equilibrium defects, samples were annealed at the temperatures of $500^{\circ} \mathrm{C}$ and $1100^{\circ} \mathrm{C}$ in vacuum $\left(p<10^{-3}\right.$ mbar) for 3 hours and then slowly cooled down to room temperature at the rate of $20^{\circ} \mathrm{C} / \mathrm{h}$. Then for the annealed samples, positron lifetimes were measured.

For further research, samples were placed between the poles of a magnet perpendicular or parallel to the magnetic induction vector. Next, the samples, appropriately oriented relative to the magnetic field, were placed in a container above the surface of a $3 \% \mathrm{HCl}$ solution, where they corroded in the vapor from this solution. Some samples were subjected to corrosion directly after annealing, without being held in a magnetic field. The magnetic field had the induction of $B=276 \mathrm{mT}$. In order to determine the distribution of defects caused by corrosion after it ended and after the positron lifetime spectrum was measured, the surface layers were removed from titanium samples by pickling them in a mixture of the hydrofluoric acid and nitric acid. Then, the samples were cleaned in ethyl alcohol and dried at the temperature of $60^{\circ} \mathrm{C}$ in a vacuum. The thickness of the removed layers was determined based on the difference between the sample mass before and after the layer was removed. Positron lifetime spectra for titanium samples described in the following were examined:

(A) annealed at the temperatures of $500^{\circ} \mathrm{C}$ and $1100^{\circ} \mathrm{C}$

(B) corroding directly after being annealed for $150 \mathrm{~h}$ outside a magnetic field;

(C) corroding directly after being annealed for $150 \mathrm{~h}$ in an external magnetic field perpendicular to the corroding surface;

(D) corroding directly after being annealed for $150 \mathrm{~h}$ in an external magnetic field parallel to the corroding surface;

(E) held without corrosion atmosphere for $150 \mathrm{~h}$ in an external magnetic field perpendicular to the sample surface;

(F) held without corrosion atmosphere for $150 \mathrm{~h}$ in an external magnetic field parallel to the sample surface.

Measurements of positron lifetime spectra was performed using a convectional fast-fast coincidence spectrometer with a time resolution $\sim 270$ ps full width of half maximum (FWHM). The obtained spectra always contained more than $10^{6}$ counts and were analyzed by decomposition them into two and three components. It was found that all the analyzed spectra contained at least two components with the following lifetimes: $\tau_{1}=(0.147 \pm 0.03) \mathrm{ns}$ and $\tau_{2}=(0.240 \pm 0.12) \mathrm{ns}$.

\section{Results}

Figure 1a shows the relationship between the intensity $I_{2}$ of the defect component of the positron lifetime spectrum with the value $\tau_{2}=0.240 \mathrm{~ns}$ and the thickness $Z$ of the removed surface layer for sample B. From the literature, it is known that the $\tau_{2}$ value corresponds to the positron lifetime in vacancies in Ti. The point marked $A$ represents samples examined directly after annealing (with an equilibrium concentration of vacancies). The values of $I_{2}$ are, within the margin of error, the same for both types of samples, but after 150 hours of corrosion, $I_{2}$ increases about 2.5 times for samples annealed at $500^{\circ} \mathrm{C}$ and almost 4 times for those annealed at $1100^{\circ} \mathrm{C}$. For samples annealed at $1100^{\circ} \mathrm{C}$, after a layer of $6.4 \mathrm{~mm}$ thickness is removed, $I_{2}$ decreases to about $8 \%$ and remains the same (within the margin of error) to the depth of $45.9 \mathrm{~mm}$. However, it remains much higher than that on the surfaces of samples examined directly after their annealing. In samples annealed at $500^{\circ} \mathrm{C}$, the intensity $I_{2}$ decreases practically down to the depth of $45 \mathrm{~mm}$, at which it reaches a value close to that on the surface of the annealed sample. The intensity $I_{2}$ is con- 

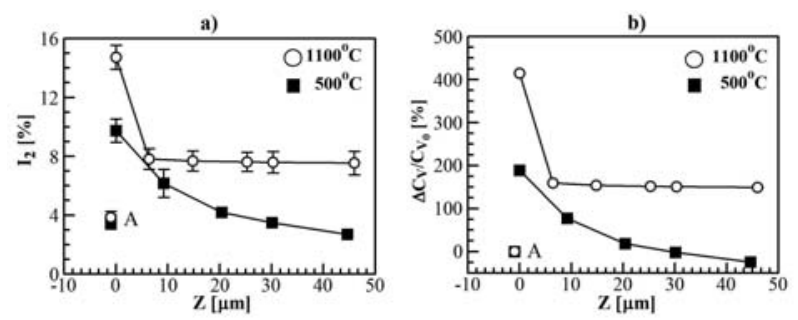

Fig. 1. Dependences of the intensity $I_{2}$ (a) and $\Delta C_{v} / C_{v o}$ (b) on the thickness $Z$ of the removed layer. Points marked $A$ apply to annealed titanium.

nected with the concentration of the defects in the area in which the positrons annihilate by relation (1). Positron lifetime measurements, depending on the thickness $Z$ of the removed surface layer of the samples, indicated that the value of component $\tau_{2}=0.240$ ns does not change, which testifies to the absence of clustering of vacancies. Figure $1 \mathrm{~b}$ shows relative changes in the vacancy concentration $\Delta C_{z} / C_{v o}$ in titanium depending on $Z\left(C_{v o}\right.$ is the vacancy concentration in non-defected titanium). Holding samples in $\mathrm{HCl}$ vapor for $150 \mathrm{~h}$ causes additional vacancies to be created by chemical reactions in the near-surface layer of about $45 \mu \mathrm{m}$ thickness. Their concentration is greater in samples annealed at the temperature of $1100^{\circ} \mathrm{C}$ than that in those annealed at $500^{\circ} \mathrm{C}$ (about 1.5 times).

To better understand the influence of an external magnetic field on the passivating layer, positron lifetime spectra were studied in titanium samples kept in a magnetic field, in atmospheric air, and at room temperature, that is, without a produced corrosive atmosphere. In such conditions, the passive layer is stable and its thickness and defective state does not change over time. Figure 2 shows diagram of the intensity $I_{2}$ for titanium samples E and F. For comparison, the figure also shows the values of these parameters before samples were placed in the magnetic field $(\mathrm{A})$. One can see that a perpendicular magnetic field (E) causes (within the margin of error) no change to $I_{2}$. However, if the magnetic field is parallel to the corroding surface $(\mathrm{F})$, after $150 \mathrm{~h}$, the values of that parameter clearly rise to a certain level which is practically independent of the temperature at which the samples were held. These results seem to prove that forces of magnetic field

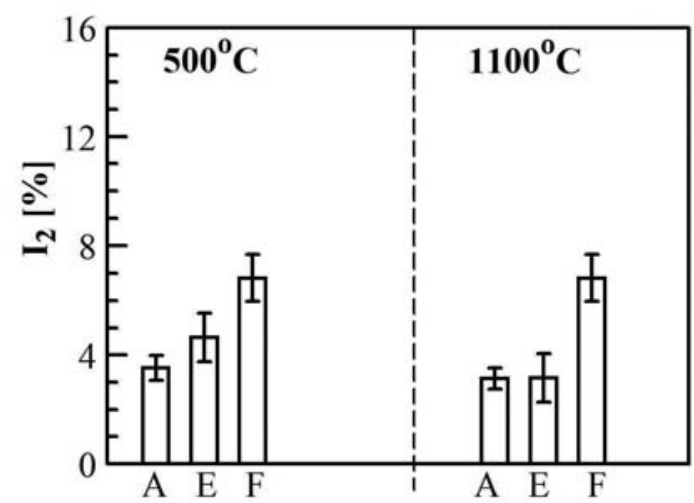

Fig. 2. Diagram of $I_{2}$ for annealed samples (A) and samples corroding in air for $150 \mathrm{~h}$ in an external magnetic field perpendicular $(\mathrm{E})$ and parallel $(\mathrm{F})$ to the corroding surface.
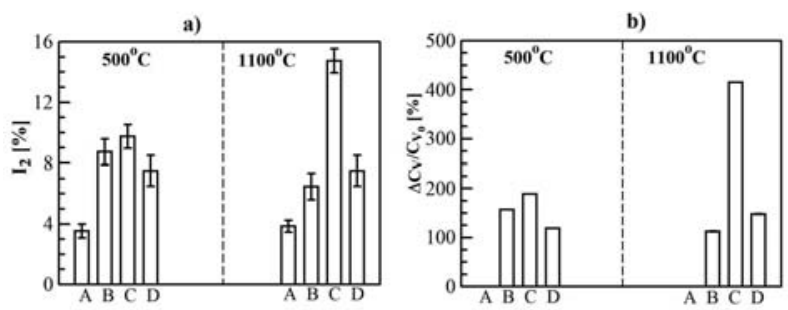

Fig. 3. Diagrams of (a) $I_{2}$ and (b) $\Delta C_{v} / C_{v o}$ determined for annealed samples (A), samples corroding for $150 \mathrm{~h}$ outside a magnetic field (B), and samples corroding for $150 \mathrm{~h}$ in a magnetic field perpendicular (C) and parallel (D) to the corroding surface.

parallel to the passivating layer tears it apart during the transport of cation and anion vacancies, which is the cause of the formation of defects. When the passive layer is in the $\mathrm{HCl}$ vapors formed in there, its mechanical properties are changed as a result of extra defects caused.

Our previous research on the influence of the magnetic field on the corrosion of ferromagnetic iron and the St20 steel has shown that during corrosion in $\mathrm{HCl}$ vapor, vacancies form clusters, whereas the kinetics of these changes depends on the orientation of the magnetic field relative to the corroded surface and on the previous magnetization of the sample before it corrodes [4, 8]. However, this research has not allowed us to draw unambiguous conclusions whether the kinetics of corrosion in a magnetic fields is influenced by the change of the passivating surface or the change of the energy state of ferromagnetic domains of the metal. Titanium is a paramagnetic material that, when held in a magnetic field, does not form or reorient magnetic domains. Diagrams in Fig. 3 shows the intensity $I_{2}$ (a) and $\Delta C_{v} / C_{v o}$ (b) measured for samples: $\mathrm{A}, \mathrm{B}, \mathrm{C}$, and $\mathrm{D}$. It can be observed that the values of $I_{2}$ and $\Delta C_{v} / C_{v o}$ are greater for all corroded samples than for non-corroded samples. The influence of the magnetic field orientation is exhibited to a greater extent in samples annealed at $1100^{\circ} \mathrm{C}$. If the magnetic field is perpendicular to the sample surface $(\mathrm{C})$, the values of $I_{2}$ and $\Delta C_{v} / C_{v_{0}}$ are greater than those for samples corroding outside a magnetic field (B) or in one that is parallel to the sample D. Within the margin of error, the changes in $I_{2}$ for samples corroding outside a magnetic field or in a magnetic field parallel to the corroding surfaces are the same.

\section{Conclusions}

1. At an early stage of corrosion in $\mathrm{HCl}$ vapor, cation vacancies form in titanium.

2. An increased concentration of vacancies formed during corrosion is found down to the depth of approx. $45 \mu \mathrm{m}$ and the distribution of their concentration depends on the temperature at which the samples were preliminarily annealed.

3. The orientation of the external magnetic field impacts differently on the kinetics of cationic vacancies formation in the uncorroded and corroded passive layer. 


\section{References}

1. Hautojärvi, P. (1979). Introduction of positron annihilation. In P. Hautojärvi (Ed.), Positron in solids (pp. 1-22). Berlin: Springer-Verlag.

2. Pietrzak, R., Szatanik, R., \& Szuszkiewicz, M. (1999). Measurements of positrons lifetimes in corroded nickel. Acta Phys. Pol. A, 95(4), 647-651.

3. Pietrzak, R., Szatanik, R., \& Jaworska, A. (2006). The influence of magnetic field on annihilation of positrons in corroded steel St-20. Acta Phys. Pol. A, 110(5), 677-681.

4. Pietrzak, R., \& Szatanik, R. (2010). Effect of magnetic field on the corrosion of iron and St20 steel as studied by positron annihilation. Phys. Status Solidi B, 247(7), 1822-1828. DOI: 10.1002/pssb.200945124.

5. Waskaas, M. (1996). Magnetic field effect on electrode reactions. I. Effects on the open-circuit potential of electrodes in solutions of different magnetic properties. Acta Chem. Scand., 50, 516-520.

6. Chiba, A., Kawazu, K., Nakano, O., Tamura, T., Yoshihara, S., \& Sato, E. (1994). The effects of mag- netic fields on the corrosion of aluminium sodium chloride solutions. Corros. Sci., 36(3), 539-543. DOI: 10.1016/0010-938X(94)90042-6.

7. Kelly, E. J. (1977). Magnetic field effects electrochemical reactions occurring at metal/flowing-electrolyte interfaces. J. Electrochem. Soc., 124(7), 987-994. DOI: $10.1149 / 1.2133514$.

8. Pietrzak, R., \& Szatanik, R. (2014). The influence of a magnetic field on the formation of corrosion defects in selected metals and steels, analyzed using positron annihilation method. Acta Phys. Pol. A, 125(3), 733-736.

9. Macdonald, D. D. (1999). Passivity - the key to our metals-based civilization. Pure Appl. Chem., 77(6), 951-978. DOI: 10.1351/pac199971060951.

10. Linhardt, P., Ball, G., \& Schlemmer, E. (2005). Electrochemical investigation of chloride induced pitting of stainless steel under the influence of a magnetic field. Corr. Sci., 47(7), 1599-1603. DOI: 10.1016/j. corsci.2004.09.002. 